\title{
Association of Eating Alone with Depressive Symptoms and Suicidal Ideation among Korean Adults
}

\author{
Joonyoung Park', Gyeongsil Lee ${ }^{1,2, *}$ \\ 'Department of Family Medicine, Seoul National University Hospital, Seoul, Korea \\ ${ }^{2}$ Department of Biomedical Sciences, Seoul National University Graduate School, Seoul, Korea
}

\begin{abstract}
Background: In recent years, eating dinner alone has become a rising social issue in Korea. Depression is one of the primary health problems that can lead to numerous negative consequences. However, few studies have focused on people who eat alone and the effect of eating alone on depression. We investigated the association between eating dinner alone and depression.

Methods: Our cross-sectional study included data from 14,093 Korean adults aged above 19 years old; data were sourced from the Korea National Health and Nutrition Examination Survey in 2014, 2016, and 2018, when the Patient Health Questionnaire (PHQ-9) was used. We classified participants based on their dinner habits: eating alone or social eating. Depression and suicidal ideation among participants were measured using the PHQ-9. Multivariate logistic regression analysis was used to investigate whether eating alone was related to depression or suicidal ideation after adjusting for age, sex, household income, education, alcohol, smoking, exercise, frequency of eating out, and living arrangement.

Results: Individuals who ate dinner alone (22.9\%) had higher depression and suicidal ideation rates than those who ate with others. Those who ate alone had greater odds ratios (ORs) of depressive symptoms (depression: OR, 1.42; 95\% confidence interval [CI], 1.27-1.58; suicidal ideation: OR, 1.49; 95\% CI, 1.25-1.78) after adjustment for covariates. The subgroup analysis shows that the odds of suicidal ideation among individuals who eat alone were nonexistent among those who performed regular aerobic exercise.

Conclusion: Eating dinner alone is closely associated with depressive symptoms, particularly suicidal ideation. Therefore, providing opportunities to eat with others may be effective for maintaining the mental health of adults.
\end{abstract}

Keywords: Depression; Suicidal Ideation; Meals; Feeding Behavior

Received: June 2, 2020, Revised: September 7, 2020, Accepted: September 9, 2020

${ }^{*}$ Corresponding Author: Gyeongsil Lee https://orcid.org/0000-0003-1910-9658

Tel: +82-2-2072-0714, Fax: +82-2-766-3276, E-mail: tint0305@snu.ac.kr; 83102@snuh.org 


\section{INTRODUCTION}

Single-person households in Korea have increased rapidly from $15.5 \%$ in 2000 to $29.3 \%$ in 2018, according to Statistics Korea. ${ }^{1)}$ This increase is related to changing social structures, such as rising marriage age, divorce rates, and bereavement caused by longer life expectancy. As a result, the number of individuals eating alone is also steadily increasing. ${ }^{2,3)}$ Individuals who eat together receive social support during shared mealtimes; therefore, eating alone may have some negative psychological impacts. ${ }^{4)}$ However, studies related to eating alone have primarily focused on the nutritional factors associated with nutritional imbalance, low food diversity, and obesity. ${ }^{5,6)}$

Few studies have examined the association between eating alone and mental health, including happiness or depressive mood, which have some limitations in the unvalidated questionnaire on mental health and small population. ${ }^{7,8)}$ Additionally, not only depressive mood but also suicidal ideation can serve as key markers for mental health, which is assessed in the ninth question of the Patient Health Questionnaire (PHQ-9). ${ }^{9)}$ Therefore, we aimed to examine the association of eating alone with depressive mood and suicidal ideation. Among the three meals eaten in a day, we examine dinner because breakfast is usually irregular, eaten at home, or skipped altogether, and lunch is often eaten in a group due to external activities, such as work.

\section{METHODS}

\section{Study Population}

This study used data from the 2014, 2016, and 2018 instances of the PHQ-9 survey during the sixth (2013-2015) and seventh (2016-2018) waves of the Korea National Health and Nutrition Examination Survey. The total number of individuals surveyed was 23,692 with 18,636 adults aged 19 years or older. Among the 18,636 participants, the exclusion criteria included (1) missing response to the question about whether dinners were eaten accompanied by others in the past year $(n=2,335),(2)$ eating-out frequency of less than twice a week $(n=347)$, and (3) missing responses on the PHQ-9 $(n=1,861)$. A total of 14,093 individuals (5,826 men and 8,267 women) were included for analysis. The procedures followed were in accordance with the ethical standards of the Institutional Review Board of the Korea Disease Control and Prevention Agency and with the Helsinki Declaration of 1975, as revised in 2000. The Institutional Review Board of the Korea Disease Control and Prevention Agency reviewed and approved the KNHANES (IRB no., 2013-12EXP-03-5C, 2018-01-03-P-A). All participants provided written informed consent.

\section{Study Variables}

We defined eating alone as the "no" response to "whether dinners were accompanied by family and non-family members" during the past year. Depression was measured using the PHQ-9. The survey comprised nine questions, which participants were asked to evaluate using a 4-point Likert scale ( $0=$ "not entirely" $1=$ "for many days"
$2=$ "more than a week" and 3="almost every day" for each question). The total score ranged from 0 to 27 points with a higher total score indicating more severe depression symptoms. In this study, participants with a total score of 5 or more points from the PHQ- 9 were defined as depressed and may thus be screened for a depressive disorder and considered for further examinations. Additionally, in the case of question 9-“How often did I experience self-harm thoughts, such as 'I would rather die' or other such thoughts, in the last 2 weeks" - the group was classified as suicidal if individuals answered anything except "not at all."

Participants' ages were classified into three variable groups: 19-39, 40-64, and 65 years old. Household type was classified into living alone and multiple-person households. Moreover, income levels were determined by dividing the participants into fourth-percentile household income variables. Education level was classified as $\leq$ middle school, high school, and $\geq$ college. Body mass index (BMI) was classified as underweight $\left(<18.5 \mathrm{~kg} / \mathrm{m}^{2}\right)$, normal $\left(18.5-22.9 \mathrm{~kg} / \mathrm{m}^{2}\right)$, overweight (23-24.9 $\left.\mathrm{kg} / \mathrm{m}^{2}\right)$, and obese $\left(\geq 25 \mathrm{~kg} / \mathrm{m}^{2}\right)$, according to the World Health Organization's obesity standards. ${ }^{10)}$ Health behaviors included smoking, alcohol consumption, and exercise. Regarding alcohol consumption, participants were defined as problematic drinkers if they drank more than twice a week with an average alcohol consumption of 7 drinks or more for men and 5 drinks or more for women; a moderate drinker was defined as a non-problematic drinker. Smoking was classified in the following categories: nonsmokers smoked less than 5 packs of cigarettes in their lifetime, ex-smokers had smoked in the past but did not currently smoke, and current smokers were smoking at the time of the survey. Aerobic exercise utilized the rate of aerobic physical activity. "Yes" and "no" were used depending on whether physical activity was performed over 2 hours and 30 minutes per week or for more than 1 hour and 15 minutes at a high-intensity level. The diet survey examined the frequency of eating out. Frequency of eating out was classified as "rarely" (not more than 3 times a month), "usually" (1-4 times a week), and "often" (more than 5 times a week).

\section{Statistical Analysis}

The chi-square test was conducted to compare general characteristics, diet, and health behaviors based on the presence or absence of dinner companions, and the continuous data, age, and BMI were analyzed using a t-test. A multivariate logistic regression analysis was conducted to determine the association between eating alone and depression. Model I did not feature adjusted variables; Model II was adjusted for gender, age, income, and education level; and Model III was adjusted for alcohol, smoking, aerobic exercise, frequency of eating out, and living arrangement in addition to factors for Model II. Statistical analysis used the software STATA ver. 16.0 (Stata Corp., College Station, TX, USA) and $\mathrm{P}<0.05$ was considered statistically significant.

\section{RESULTS}

The basic characteristics, based on the presence or absence of dinner 
Table 1. General characteristics of subject by dinner companion $(n=14,093)$

\begin{tabular}{|c|c|c|c|c|}
\hline \multirow{2}{*}{ Characteristic } & \multirow{2}{*}{ Eating alone } & \multicolumn{2}{|c|}{ Eating together } & \multirow{2}{*}{ P-value } \\
\hline & & With family & With non-family & \\
\hline No. of subjects & 3,223 & 9,280 & 1,590 & \\
\hline Age (y) & $56.56 \pm 17.09$ & $52.08 \pm 15.94$ & $42.28 \pm 14.95$ & $<0.001$ \\
\hline Male & 35.09 & 60.06 & 40.30 & $<0.001$ \\
\hline Body mass index $\left(\mathrm{kg} / \mathrm{m}^{2}\right)$ & $23.97 \pm 3.56$ & $23.80 \pm 3.47$ & $24.13 \pm 3.57$ & \\
\hline Household income & & & & $<0.001$ \\
\hline Lower half & 57.69 & 42.16 & 27.88 & \\
\hline Upper half & 42.31 & 57.84 & 72.12 & \\
\hline Education level & & & & $<0.001$ \\
\hline Middle school (grade 6-9) or lower & 44.71 & 28.98 & 12.96 & \\
\hline High school (grade 10-12) & 23.66 & 28.16 & 23.98 & \\
\hline College or higher & 31.64 & 42.86 & 63.06 & \\
\hline Alcohol & & & & $<0.001$ \\
\hline Moderate drinking & 86.97 & 85.51 & 71.89 & \\
\hline Problem drinking & 13.03 & 14.49 & 28.11 & \\
\hline Smoking & & & & $<0.001$ \\
\hline Never & 67.66 & 65.11 & 51.17 & \\
\hline Ex & 16.00 & 20.42 & 18.28 & \\
\hline Current & 16.34 & 14.46 & 30.55 & \\
\hline Aerobic exercise & & & & $<0.001$ \\
\hline Yes & 44.37 & 44.93 & 50.94 & \\
\hline No & 55.63 & 55.07 & 49.06 & \\
\hline Eating out frequency & & & & $<0.001$ \\
\hline Rare (s3 times/mo) & 35.56 & 29.89 & 5.60 & \\
\hline Usually (1-4 times/wk) & 35.15 & 38.08 & 14.47 & \\
\hline Often ( $\geq 5$ times/wk) & 29.29 & 32.03 & 79.94 & \\
\hline Living alone (\%) & & & & $<0.001$ \\
\hline Yes & 39.50 & 1.25 & 16.73 & \\
\hline No & 60.50 & 98.75 & 83.27 & \\
\hline Depression* & 26.62 & 17.67 & 18.36 & $<0.001$ \\
\hline Suicidal ideation ${ }^{\dagger}$ & 10.98 & 5.24 & 4.65 & $<0.001$ \\
\hline
\end{tabular}

Values are presented as mean \pm standard deviation or \%, unless otherwise stated. P-values by chi-square test or t-test. Eating alone: do not have accompanied family members and non-family members at dinner for the past year; household income: monthly income of the householder of the year is divided into two categories; moderate drinking: non-problem drinking; problem drinking: drink more than twice a week and the average alcohol consumption is 7 drinks or more for men and 5 drinks or more for women; never smokers: less than five packs of cigarettes in their lifetime; ex-smokers: smoked in the past but did not currently smoke; aerobic exercise: and physical activity was performed over 2 hours and 30 minutes per week, or for more than 1 hour and 15 minutes at a high-intensity level.

PHQ, Patient Health Questionnaire.

${ }^{*} \mathrm{PHQ}-9 \geq 5$. ${ }^{\mathrm{P} H Q}-9$ 9th question.

Table 2. OR for depression or suicidal ideation of eating alone individuals compare to eating together individuals

\begin{tabular}{lcccc}
\hline \multirow{2}{*}{ Variable } & Eating together & \multicolumn{3}{c}{ OR (95\% Cls) of eating alone individuals } \\
\cline { 3 - 5 } & 1 & Model I & Model II & Model III \\
\hline Depression & 1 & $1.68(1.53-1.84)$ & $1.48(1.34-1.63)$ & $1.42(1.27-1.58)$ \\
Suicidal ideation & $2.27(1.98-2.61)$ & $1.61(1.39-1.87)$ & $1.49(1.25-1.78)$ \\
\hline
\end{tabular}

Model I: unadjusted; model II: adjusted for sex, age, income, and education; and model III: adjusted for alcohol, smoking, aerobic exercise, eating out frequency, and living arrangement in addition to model II.

$\mathrm{OR}$, odds ratio; $\mathrm{Cl}$, confidence interval.

companions, are presented in Table 1. Individuals who ate alone accounted for $29.7 \%$ with both men and women tending to be older, have lower income, and have lower education levels. In the eating alone group, the frequency of eating out was significantly lower. Moreover, $35.8 \%$ individuals stated that they had not drunk alcohol in the past year. The eating alone group had a higher rate of depression (a PHQ-9 score of 5 or higher) than the eating together group. Addition- ally, among the eating alone group, the proportion of those with suicidal ideations was significantly higher than for the social eating group. In the eating together group, most people reported that they dined with their families. Individuals who ate with family members accounted for $85.4 \%$, thereby indicating a tendency to be older, with a higher percentage of men and lower frequency of eating out.

Table 2 shows that the relationship of depression or suicidal ideation 
depends on the presence or absence of dinner companions. The analysis results for model I, which was unadjusted for other variables, showed that eating alone was $68 \%$ more likely to cause depression, and suicidal ideations were 2.27 times more likely than for social eaters. Model II was adjusted for sex, age, income, and education and showed 1.48 times higher depression levels and 1.61 times higher suicidal ideation levels among the eating alone group. In addition to model II, model III was adjusted for alcohol, smoking, aerobic exercise, frequency of eating out, and living arrangements. The eating alone group was 1.42 times more depressed and 1.49 times more prone to suicidal ideation. Table 3 indicates whether the dinner companion is or is not a family member and shows that the difference between family and non-family groups is insignificant. Therefore, in both groups, the association was lower than in the eating alone group.

Table 4 presents the subgroup analysis, and the trend is similar to the primary results. Risk factors for depression and suicidal ideation included young age, male sex, low BMI, low income, low education level, problematic drinking, and being a current smoker. In particular, the risk of suicidal ideation was higher among older individuals. The

Table 3. Odds ratio for depression or suicidal ideation between groups depending on the members of dinner companion

\begin{tabular}{lccc}
\hline \multicolumn{1}{c}{ Variable } & Eating alone & With family & With non-family \\
\hline Depression & & & \\
Model I & 1 & $0.59(0.54-0.65)$ & $0.62(0.53-0.72)$ \\
Model II & 1 & $0.67(0.60-0.74)$ & $0.75(0.64-0.88)$ \\
Model III & 1 & $0.70(0.62-0.78)$ & $0.74(0.63-0.88)$ \\
Suicidal ideation & & & \\
Model I & 1 & $0.45(0.39-0.52)$ & $0.40(0.31-0.51)$ \\
Model II & 1 & $0.61(0.53-0.71)$ & $0.71(0.54-0.93)$ \\
Model III & 1 & $0.66(0.55-0.79)$ & $0.71(0.54-0.94)$ \\
\hline
\end{tabular}

Values are presented as odds ratio ( $95 \%$ confidence interval). Model I: unadjusted; model II: adjusted for sex, age, income, and education; and model III: adjusted for alcohol, smoking, aerobic exercise, eating out frequency, and living arrangement in addition to model II.

Table 4. Subgroup analysis of the association between types of dinner companion and depression or suicidal ideation

\begin{tabular}{|c|c|c|c|c|}
\hline \multirow[b]{2}{*}{ Variable } & \multicolumn{2}{|r|}{ Depression } & \multicolumn{2}{|c|}{ Suicidal ideation } \\
\hline & $\begin{array}{l}\text { No. of depression/no. of } \\
\text { subtotals }\end{array}$ & $\begin{array}{l}\text { OR }(95 \% \mathrm{Cl}) \text { of eating alone individuals } \\
\text { compared with eating together }\end{array}$ & $\begin{array}{l}\text { No. of suicidal ideation/no. of } \\
\text { subtotals }\end{array}$ & $\begin{array}{l}\text { OR ( } 95 \% \mathrm{Cl} \text { ) of eating alone individuals } \\
\text { compared with eating together }\end{array}$ \\
\hline \multicolumn{5}{|l|}{ Age $(y)$} \\
\hline $19-39$ & $872 / 3,797$ & $1.51(1.22-1.87)$ & $172 / 3,797$ & $1.62(1.09-2.39)$ \\
\hline $40-64$ & $1,107 / 6,491$ & $1.39(1.18-1.64)$ & $353 / 6,491$ & $1.37(1.04-1.79)$ \\
\hline$\geq 65$ & $811 / 3,805$ & $1.39(1.12-1.74)$ & $389 / 3,805$ & $1.58(1.17-2.12)$ \\
\hline \multicolumn{5}{|l|}{ Sex } \\
\hline Men & $820 / 5,826$ & $1.55(1.27-1.88)$ & $277 / 5,826$ & $1.80(1.32-2.46)$ \\
\hline Women & $1,970 / 8,267$ & $1.37(1.20-1.57)$ & $637 / 8,267$ & $1.38(1.11-1.71)$ \\
\hline \multicolumn{5}{|l|}{ Body mass index } \\
\hline Underweight & $180 / 559$ & $1.57(0.95-2.56)$ & $57 / 559$ & $0.94(0.42-2.13)$ \\
\hline Normal & $1,701 / 8,719$ & $1.45(1.26-1.67)$ & $547 / 8,719$ & $1.57(1.26-1.96)$ \\
\hline Overweight & $900 / 4,772$ & $1.33(1.09-1.62)$ & $306 / 4,772$ & $1.51(1.11-2.06)$ \\
\hline \multicolumn{5}{|l|}{ Household income } \\
\hline Lower half & $1,510 / 6,199$ & $1.48(1.27-1.74)$ & $622 / 6,199$ & $1.49(1.19-1.86)$ \\
\hline Upper half & $1,271 / 7,860$ & $1.36(1.16-1.59)$ & $289 / 7,860$ & $1.49(1.12-2.00)$ \\
\hline \multicolumn{5}{|l|}{ Education level } \\
\hline Middle school or lower & $1,030 / 4,334$ & $1.55(1.28-1.86)$ & $492 / 4,334$ & $1.51(1.17-1.94)$ \\
\hline High school & $712 / 3,755$ & $1.18(0.95-1.47)$ & $201 / 3,755$ & $1.23(0.84-1.79)$ \\
\hline College or higher & $1,048 / 5,997$ & $1.51(1.26-1.80)$ & $219 / 5,997$ & $1.78(1.27-2.48)$ \\
\hline \multicolumn{5}{|l|}{ Alcohol } \\
\hline Moderate drinking & $2,426 / 12,391$ & $1.38(1.23-1.56)$ & $788 / 12,391$ & $1.47(1.21-1.78)$ \\
\hline Problem drinking & $348 / 1,642$ & $1.72(1.30-2.29)$ & $119 / 1,642$ & $1.75(1.14-2.70)$ \\
\hline \multicolumn{5}{|l|}{ Smoking } \\
\hline Never & $1,836 / 8,993$ & $1.31(1.15-1.50)$ & $573 / 8,993$ & $1.47(1.18-1.83)$ \\
\hline Ever & $935 / 5,032$ & $1.66(1.37-2.01)$ & $331 / 5,032$ & $1.53(1.14-2.07)$ \\
\hline \multicolumn{5}{|l|}{ Aerobic exercise } \\
\hline No & $1,564 / 7,658$ & $1.52(1.31-1.77)$ & $555 / 7,658$ & $1.69(1.34-2.12)$ \\
\hline Yes & $1,212 / 6,389$ & $1.30(1.10-1.53)$ & $352 / 6,389$ & $1.25(0.94-1.65)$ \\
\hline \multicolumn{5}{|l|}{ Eating out frequency } \\
\hline Rare & $945 / 4,009$ & $1.61(1.33-1.96)$ & $431 / 4,009$ & $1.29(0.97-1.70)$ \\
\hline More than once a week & $1,845 / 10,084$ & $1.35(1.18-1.54)$ & $483 / 10,084$ & $1.65(1.31-2.07)$ \\
\hline \multicolumn{5}{|l|}{ Living alone } \\
\hline Yes & $479 / 1,655$ & $1.34(0.99-1.80)$ & $229 / 1,655$ & $1.59(1.01-2.50)$ \\
\hline No & $2,311 / 12,438$ & $1.43(1.27-1.61)$ & $685 / 12,438$ & $1.48(1.22-1.79)$ \\
\hline
\end{tabular}

$\mathrm{OR}$, odds ratio; $\mathrm{Cl}$, confidence interval. 
risk of depression was higher in the group that did not exercise; however, no relevant correlation was noted between suicidal ideation and exercise level.

\section{DISCUSSION}

Eat alone was associated with higher depression symptoms, including suicidal ideation. Individuals who ate dinner alone were older, had lower income and education level, and were more likely to be in single-person households. Moreover, frequency of eating out was lower in the eating alone group. When comparing family and non-family groups, the difference was not significant, and, compared to groups eating alone, both groups showed lower association. Additionally, a subgroup analysis showed that, compared with the counter subgroups, the magnitude of depressive mood and suicidal ideation was higher in the subgroup including young adults aged 19-39 years, adult men, individuals with lower income, individuals with low education levels, individuals engaging in risky alcohol consumption, individuals who had smoked in their lifetime, underweight individuals, and individuals who did not engage in exercise.

Our findings are consistent with those of previous studies that eating alone can be a risk factor for depression, though most such studies focused on older adults. ${ }^{3,8,11,12)}$ For older people, eating is one of the most important social activities, and they can gain social interactions through meals with others. ${ }^{4)}$ We found that, not only for older adults but also for young adults, eating alone was associated with depressive mood and suicidal ideation; the odds ratio was higher for this group than for middle-aged adults. A recent study examined the perceptions of commensality and solo-eating among Korean and Japanese college students and found that Korean college students tended to adopt a negative perspective of eating alone and preferred commensality. ${ }^{2)}$ Communication through shared meals with family members or friends is related to appetite or depression, particularly emotional support, which influences an individual's quality of life. Regarding gender, a study analyzing the association between eating alone and depression among elderly people found that, among men, eating alone had a greater effect on depression, particularly when living alone. ${ }^{11)}$ We also found that men who ate alone experienced more depression and higher suicidal ideation compared with men who ate together; the magnitude of odds ratio for men was higher, compared to that of women, thereby suggesting that eating alone had a stronger effect on men's mental health, compared with that on women's mental health. A significant difference was noted in the ratio of smokers between eating alone and social eating. Smoking involves various social factors, such as income and education levels, ${ }^{13)}$ and is closely linked to depression. ${ }^{14,15)}$ Therefore, these factors can affect the association between smoking and eating alone, and, after adjusting for the factors, smokers showed a significantly higher risk of depression and suicidal ideation than nonsmokers. For people who exercised, the odds ratio of eating alone on depression was lower than for those who did not exercise. The antidepressant effects of exercise are controversial; however, based on evidence in the literature, the preventive effects of exercise on depression have been reported in large cohort studies. ${ }^{16,17)}$ Therefore, the current study results showed preliminary evidence for the advantages of exercise for mental health. Studies have shown that BMI and depression represent inverse U-shaped curves while others suggested that individuals are more susceptible to various diseases when underweight. ${ }^{18,19)}$ Other studies have shown that underweight women are increasingly prone to diseases and depression. ${ }^{20)} \mathrm{We}$ found that the underweight group had a high risk of depression (odds ratio, 1.57). Studies have shown that eating patterns are related to practical problems, such as decreased appetite with relation to mental health. ${ }^{21)} \mathrm{A}$ meal is not only about eating; it positively affects the creation of intimacy and pleasant feelings through relationships with others. ${ }^{22)}$ Therefore, individuals can be adversely affected by eating alone-particularly Koreans, because, at a societal level, they tend to feel awkward and ashamed while eating alone.

This study had the following limitations. First, the Korea National Health and Nutrition Examination Survey is a cross-sectional survey and, therefore, cannot clarify the causality of the association between eating alone and mental health. The survey of eating habits focuses on the past year, and PHQ-9 is based on the past 2 weeks, thus ensuring reduced bias. Second, the survey specifically inquired about dinner companions; therefore, it is not entirely possible to rule out results that may have been influenced by a breakfast or lunch partner. Breakfast and lunch are often eaten simply as takeout meals or at the workplace, whereas dinner is meaningful because it is usually with eaten with others who have a special relationship with the individual, and it is a mealtime after completing the day's work. Third, there may be deviations that can be attributed to participants' memory because we used self-reported surveys about their lifestyle. Finally, some covariables could not be corrected because they were not included in the data despite affecting depression or suicidal ideation.

Despite these limitations, this study has several advantages. First, we used representative data from the Korea National Health and Nutrition Examination Survey, which examined the presence or absence of family members for shared meals only until the fifth period (2010-2012), but, at the beginning of the first year of the sixth period (2013), the survey more accurately defined eating alone/social eating by including the option for non-family partners. Second, although several studies have focused on the relationship between eating alone and depression, these studies have focused on a specific age group. Depression is not a problem for one specific age group and may be a risk for individuals of all ages. We examined these factors among adults in a wide age range. Finally, we examined suicidal ideation risk, which is a grievous depression symptom. The results indicated that individuals who eat alone have greater odds of suicidal ideation, suggesting that eating alone can not only trigger depression but also lead to serious symptoms.

Our research presented important public health implications. Eating with others led to lower odds ratio of depression. Recently, the number of individuals eating alone has been increasing due to the im- 
pacts of coronavirus disease 2019 (COVID-19), which can harm the mental health of these individuals. Mental health care should consider social relationships thoroughly by considering mealtime companionship. ${ }^{23)}$ Providing group meals can effectively maintain mental health among adults. For instance, shared meal services at community centers that allow people to eat with local residents without relying on home-delivered food may be beneficial, particularly among older adults. In clinical practice, assessing the eating environment can be valuable for early identification of a depressed person at high risk for suicidal ideation. Older adults and women are generally considered vulnerable to mental health problems; however, younger adults or men who eat alone must also undergo mental health examinations. As mentioned earlier, this cross-sectional study cannot clarify the causality between eating alone and depression. However, as society becomes increasingly individualized, eating alone is widely accepted, and, recently, social separation has been encouraged, particularly due to the impacts of COVID-19, leading to potential harm to individuals' mental health. Further investigation, such as a prospective cohort study, is needed to confirm whether eating alone affects depression symptoms and interventions for increasing social eating frequency can alleviate depression in individuals who typically eat alone. ${ }^{24)}$

\section{CONFLICT OF INTEREST}

No potential conflict of interest relevant to this article was reported.

\section{ORCID}

Joonyoung Park: https://orcid.org/0000-0002-7324-5813

Gyeongsil Lee: https://orcid.org/0000-0003-1910-9658

\section{REFERENCES}

1. Statistics Korea. Single-person households in Korea [Internet]. Daejeon: Statistics Korea; 2020 [cited 2020 Apr 23]. Available from: http:// kosis.kr/statHtml/statHtml.do?orgId=101\&tblId=DT_1YL21161\&co nn_path=I2.

2. Cho W, Takeda W, Oh Y, Aiba N, Lee Y. Perceptions and practices of commensality and solo-eating among Korean and Japanese university students: a cross-cultural analysis. Nutr Res Pract 2015;9:523-9.

3. Kimura Y, Wada T, Okumiya K, Ishimoto Y, Fukutomi E, Kasahara Y, et al. Eating alone among community-dwelling Japanese elderly: association with depression and food diversity. J Nutr Health Aging 2012;16: 728-31.

4. Vesnaver E, Keller HH. Social influences and eating behavior in later life: a review. J Nutr Gerontol Geriatr 2011;30:2-23.

5. Locher JL, Robinson CO, Roth DL, Ritchie CS, Burgio KL. The effect of the presence of others on caloric intake in homebound older adults. J Gerontol A Biol Sci Med Sci 2005;60:1475-8.

6. Hammons AJ, Fiese BH. Is frequency of shared family meals related to the nutritional health of children and adolescents? Pediatrics 2011; 127:e1565-74.
7. Yiengprugsawan V, Banwell C, Takeda W, Dixon J, Seubsman SA, Sleigh AC. Health, happiness and eating together: what can a large Thai cohort study tell us? Glob J Health Sci 2015;7:270-7.

8. Lee SA, Park EC, Ju YJ, Nam JY, Kim TH. Is one's usual dinner companion associated with greater odds of depression?: using data from the 2014 Korean National Health and Nutrition Examination Survey. Int J Soc Psychiatry 2016;62:560-8.

9. Choi HS, Choi JH, Park KH, Joo KJ, Ga H, Ko HJ, et al. Standardization of the Korean version of Patient Health Questionnaire-9 as a screening instrument for major depressive disorder. J Korean Acad Fam Med 2007;28:114-9.

10. Weir CB, Jan A. BMI classification percentile and cut off points [Internet]. Treasure Island (FL): StatPearls; 2020 [cited 2020 Apr 20]. Available from: http://www.ncbi.nlm.nih.gov/books/NBK541070/.

11. Tani Y, Sasaki Y, Haseda M, Kondo K, Kondo N. Eating alone and depression in older men and women by cohabitation status: the JAGES longitudinal survey. Age Ageing 2015;44:1019-26.

12. Kimura Y, Okumiya K, Sakamoto R, Ishine M, Wada T, Kosaka Y, et al. Comprehensive geriatric assessment of elderly highlanders in Qinghai, China IV: comparison of food diversity and its relation to health of Han and Tibetan elderly. Geriatr Gerontol Int 2009;9:359-65.

13. Kim SR, Kim OK, Yun KE, Khang YH, Cho HJ. Socioeconomic factors associated with initiating and quitting cigarette smoking among Korean men. Korean J Fam Med 2009;30:415-25.

14. Won MR, Ahn MS, Choi YJ. Factors associated with perceived depression of Korean adults: secondary data from the Korean Community Health Survey. Community Ment Health J 2017;53:288-96.

15. Sung HN, Kim JS. The relationship between smoking and depressive symptoms among Korean adults. Korean J Health Educ Promot 2016; 33:57-66.

16. Pascoe M, Bailey AP, Craike M, Carter T, Patten R, Stepto N, et al. Physical activity and exercise in youth mental health promotion: a scoping review. BMJ Open Sport Exerc Med 2020;6:e000677.

17. Park SC. Antidepressive effects of exercise. J Korean Neuropsychiatr Assoc 2018;57:139-44.

18. De Wit LM, van Straten A, van Herten M, Penninx BW, Cuijpers P. Depression and body mass index, a u-shaped association. BMC Public Health 2009;9:14.

19. Batty GD, Whitley E, Kivimaki M, Tynelius P, Rasmussen F. Body mass index and attempted suicide: cohort study of 1,133,019 Swedish men. Am J Epidemiol 2010;172:890-9.

20. Helgstrand S, Andersen AM. Maternal underweight and the risk of spontaneous abortion. Acta Obstet Gynecol Scand 2005;84:1197-201.

21. Engel JH, Siewerdt F, Jackson R, Akobundu U, Wait C, Sahyoun N. Hardiness, depression, and emotional well-being and their association with appetite in older adults. J Am Geriatr Soc 2011;59:482-7.

22. Danesi G. Pleasures and stress of eating alone and eating together among French and German young adults. MENU 2012;1:77-91.

23. Kuroda A, Tanaka T, Hirano H, Ohara Y, Kikutani T, Furuya H, et al. Eating alone as social disengagement is strongly associated with depressive symptoms in japanese community-dwelling older adults. J Am Med Dir Assoc 2015;16:578-85.

24. Choi MJ, Park YG, Kim YH, Cho KH, Nam GE. Eating together and health-related quality of life among Korean adults. J Nutr Educ Behav 2020;52:758-65. 